\title{
Dynamic aperture evaluation of the proposed lattices for the RHIC 2009 polarized proton run
}

\author{
Y. Luo, M. Bai, J. Beebe-Wang, W. Fischer, C. Montag, G. Robert-Demolaize, \\ T. Satogata, S. Tepikian, D. Trbojevic
}

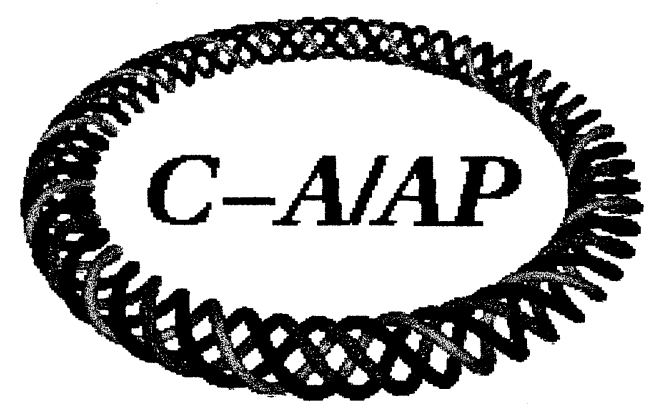

\section{Collider-Accelerator Department Brookhaven National Laboratory Upton, NY 11973}

Notice: This document has been authorized by employees of Brookhaven Science Associates, LLC under Contract No. DE-AC02-98CH10886 with the U.S. Department of Energy. The United States Government retains a nonexclusive, paid-up, irrevocable, world-wide license to publish or reproduce the published form of this document, or allow others to do so, for United States Government purposes. 
January 9,2009

\title{
Dynamic aperture evaluation of the proposed lattices for the RHIC 2009 polarized proton run
}

\author{
Y. Luo, M. Bai, J. Beebe-Wang, W. Fischer, C. Montag, \\ G. Robert-Demolaize, T. Satogata, S. Tepikian, and D. Trobjevic \\ Brookhaven National Laboratory, Upton, NY 11973, USA
}

In the article we evaluate the dynamic apertures of the proposed lattices for the coming Relativistic Heavy Ion Collider (RHIC) 2009 polarized proton (pp) $100 \mathrm{GeV}$ and $250 \mathrm{GeV}$ runs. One goal of this study is to find out the appropriate $\beta^{*}$ for the coming $2009 \mathrm{pp}$ runs. Another goal is to study the effect of second order chromaticity correction in the RHIC pp runs.

\section{Introduction}

To further increase the luminosity in the RHIC polarized proton run, we can reduce $\beta^{*}$ functions at the interaction points and increase the bunch intensity. In the previous pp runs, the $\beta^{*}$ s at IP6 and IP8 were about $0.9 \mathrm{~m}$ at store. $\beta^{*}=0.7 \mathrm{~m}$ at store has been achieved in the 2008 dedicated beam experiments. During the $2008 \mathrm{pp}$ run the bunch intensity in the Blue ring has reached a value of $1.7 \times 10^{11}$.

Reducing $\beta^{*}$ requires a large physical aperture in triplets. And a lower $\beta^{*}$ increases the sextupole correction strengths for the chromatic correction. The higher order chromaticities are also increased. To achieve acceptable beam dynamic aperture, both nonlinear chromaticity and sextupole resonance driving terms have to be well compensated.

The beam-beam tune spread is proportional to the bunch intensity. In the current RHIC pp run, the working points are constrained between $2 / 3$ and $7 / 10$. Currently the nominal uncollisional working points for the Blue and Yellow rings are set to $(28.685,29.695)$ and $(28.695,29.685$, respectively. With bunch intensity $2.0 \times 10^{11}$ or larger, there will be no enough tune space to hold the beam-beam tune spread $[1,2]$.

During the summer shutdown of RHIC 2006, the number of the power supplies for the arc chromatic sextupoles were doubled from 12 to 24 to allow correction of the second order chromaticities [3, 4]. In the RHIC pp runs, the second order chromaticity correction may mitigate the beam-beam effects with the increased bunch intensity and the reduced $\beta^{*}$ at interaction points (IPs) [5].

In the following, we first describe the lattice and beam parameters of the proposed solutions for the RHIC 2009 pp runs. Then we calculate the $10^{6}$ turn dynamic apertures with different $\beta^{*}$, bunch intensity and transverse beam emittance. In the end, the effect of second order chromaticity correction on the beam lifetime is also evaluated.

\section{Beam and optics parameters}

For all the proposed RHIC run 09 lattices, the nominal tunes are set to $(28.685,29.695)$ and $(28.695$, 29.685). The linear chromaticities are corrected to 1 . The second order chromaticities can be corrected with 8 chromatic sextupole families [3]. All the studies are based on the Blue ring lattices. The magnetic nonlinearities in the interaction regions (IRs) are included in all studied lattices.

For the $100 \mathrm{GeV}$ RHIC pp run, we will calculate the dynamic apertures for the three lattices with $\beta^{*}=0.9 \mathrm{~m}, 0.7 \mathrm{~m}$, and $0.5 \mathrm{~m}$. For the $250 \mathrm{GeV}$ RHIC pp run, we will calculate the two lattices with $\beta^{*}=0.7 \mathrm{~m}$ and $0.5 \mathrm{~m}$. The $\beta \mathrm{s}$ at other symmetric points IP10, IP12, IP2, IP4 are set to $5 \mathrm{~m}$. Table 1 lists the optics and beam parameters of the proposed lattices for the RHIC $2009100 \mathrm{GeV}$ and $250 \mathrm{GeV}$ runs.

Table 2 lists the maximum $\beta$, natural chromaticities ( without any chromaticity correction ) for the three proposed $100 \mathrm{GeV}$ pp run lattices with $\beta^{*}=0.9 \mathrm{~m}, 0.7 \mathrm{~m}$, and $0.5 \mathrm{~m}$. The uncollisional tunes are $(28.685$, 29.695). From Table 2, the maximum $\beta$ increases about $60 \%$ when $\beta^{*}$ is squeezed from $0.9 \mathrm{~m}$ to $0.5 \mathrm{~m}$. And the natural chromaticity increases by about $25 \%$. 
Table 3 lists the sextupoles correction strengths and the second and third order chromaticities after first order chromaticities are corrected with the 2 -family correction scheme. The 2-family correction scheme only corrects the first order chromaticities to +1 . The correction strengths for all focusing sextupoles (SFs) are same, while the correction strengths for all defocusing sextupoles (SDs) are same, too. From Table 3, after correcting the linear chromaticities to 1 , the required correction sextupole strengths increase about $30 \%$ when $\beta^{*}$ is squeezed from $0.9 \mathrm{~m}$ to $0.5 \mathrm{~m}$. The second order chromaticities are about 2000 to 3000 for the lattice with $\beta^{*}=0.9 \mathrm{~m}$. For the lattice with $\beta^{*}=0.5 \mathrm{~m}$, the second order chromaticities are $(-3706,6620)$, which must be corrected.

Table 1: The optics and beam parameters for the RHIC pp 09 run

\begin{tabular}{lc|c}
\hline \hline quantity & $100 \mathrm{GeV}$ & $250 \mathrm{GeV}$ \\
relativistic factor & 106 & 266 \\
normalized transverse emittances & 20 or 17 or $14 \pi \mathrm{mm} . \mathrm{mrad}$ \\
$\beta^{*}$ at IP6 and IP8 & $0.9 \mathrm{~m}$ or $0.7 \mathrm{~m}$ or $0.5 \mathrm{~m}$ & $0.7 \mathrm{~m}$ or $0.5 \mathrm{~m}$ \\
$\beta$ at other IPs & $5 \mathrm{~m}$ & $5 \mathrm{~m}$ or $7.5 \mathrm{~m}$ \\
uncollisional tunes & $(28.685,29.692)$ or $(28.695,29.685))$ \\
chromaticities & \multicolumn{2}{c|}{$(1.0,1.0)$} \\
rms longitudinal phase space area & \multicolumn{2}{c}{$0.17 \mathrm{eV} . \mathrm{s}$} \\
rms bunch length & $0.55 \mathrm{~m}$ & $0.45 \mathrm{~m}$ \\
rms relative momentum deviation & 0.00028 & 0.00014 \\
\hline \hline
\end{tabular}

\section{Second order chromaticity correction}

Second order chromaticities can be corrected with the 8 sextupole families with the Harmon module and with the 4-knob method [4]. With the Harmon module, we use 8 sextupole families or 8 variables to minimize the second and the third order chromaticities and the first order off-momentum $\beta$-beat at IP6. Harmon uses methods of general multi-dimensional nonlinear optimization to reduce the overall error function $\chi^{2}$. Most of the time the Harmon module gives very good correction results and the second order chromaticities normally are less than 50 units after corrections. However, we noticed that the Harmon module some time fails to avoid the polarity reversal of sextupole families and introduces a large unbalance in the correction strengths among the 8 families for the low $\beta^{*}$ RHIC 2009 run pp lattices.

Beside the second order chromaticity correction with the Harmon module, we propose 4 knobs corresponding to 4 chromatic sextupole families to easily and effectively minimize half-integer resonance driving terms to correct the second order chromaticities. The 4 knobs are the 4 pairs of chromatic sextupole families: ( SFPO and SFMO ), (SFPI and SFMI) ( SDPO and SDMO ) and (SDPI and SDMI). In each correction step we adjust the two families of one pair with same amount of absolute strength but with opposite signs. The advantage of the 4-knob method is that it doesn't change the first order chromaticities during the corrections of second order chromaticities. From the simulation study [4], we learn that the 4-knob method does reduce the unbalance in the correction strengths among the sextupole families and avoids the reversal of sextupole polarities. Therefore, it yields a larger dynamic aperture than the Harmon module for the low $\beta^{*}$ RHIC 2009 run pp lattices.

As an example, Table 4 shows the required sextupole correction strengths from the Harmon module and from the 4-knob method. Three proposed lattices with $\beta^{*}=0.9 \mathrm{~m}, 0.7 \mathrm{~m}$, and $0.5 \mathrm{~m}$ for the RHIC 2009 $100 \mathrm{GeV}$ pp run are used. With the 4-knob method, we normally stop the correction iterations after the second order chromaticities are corrected below 500. From the operational experience, $\xi_{x, y}^{(2)}$ below 500 are

Table 2: Lattice information for the three proposed $100 \mathrm{GeV}$ pp run lattices.

\begin{tabular}{lcc}
\hline \hline Lattice $\beta *$ & $\left(\beta_{x, \max }, \beta_{y, \max }\right)$ & $\left(\xi_{x, 0}^{(1)}, \xi_{y, 0}^{(1)}\right)$ \\
$0.9 \mathrm{~m}$ & $(1456,1484)$ & $(-79,-81)$ \\
$0.7 \mathrm{~m}$ & $(1894,1927)$ & $(-89,-92)$ \\
$0.5 \mathrm{~m}$ & $(2549,2587)$ & $(-106,-108)$ \\
\hline \hline
\end{tabular}


Table 3: Chromaticity correction with the 2-family scheme for the the three proposed $100 \mathrm{GeV}$ pp run lattices .

\begin{tabular}{lccc}
\hline \hline Lattice $\beta *$ & $\left(\left(k_{2} d l\right)_{S F},\left(k_{2} d l\right)_{S D}\right)$ & $\left(\xi_{x}^{(2)}, \xi_{y}^{(2)}\right)$ & $\left(\xi_{x}^{(3)}, \xi_{y}^{(3)}\right)$ \\
\hline $0.9 \mathrm{~m}$ & $(0.234,-0.448)$ & $(-2606,2792)$ & $(5.026 \mathrm{e} 5,2.849 \mathrm{e} 4)$ \\
$0.7 \mathrm{~m}$ & $(0.268,-0.510)$ & $(-3135,3995)$ & $(11.25 \mathrm{e} 5,11.04 \mathrm{e} 4)$ \\
$0.5 \mathrm{~m}$ & $(0.316,-0.602)$ & $(-3706,6620)$ & $(26.78 \mathrm{e} 6,32.14 \mathrm{e} 4)$ \\
\hline \hline
\end{tabular}

Table 4: Required correction strengths from the Harmon module and 4-knob method.

\begin{tabular}{lcc}
\hline \hline Sextupole Families & $\begin{array}{c}\text { Correction strengths } \\
\text { from Harmon }\end{array}$ & $\begin{array}{c}\text { Correction strengths } \\
\text { from 4-knobs }\end{array}$ \\
\hline$\beta^{*}=0.9 \mathrm{~m}$ lattice: & & \\
SFPO & 0.48 & 0.31 \\
SFMO & 0.40 & 0.31 \\
SFPI & 0.31 & 0.39 \\
SFMI & 0.07 & 0.23 \\
SDPO & -0.21 & -0.41 \\
SDMO & -0.74 & -0.78 \\
SDPI & -0.56 & -0.60 \\
SDMI & -0.88 & -0.60 \\
corrected $\xi_{x, y}^{(2)}$ & $(44,10)$ & $(-449,435)$ \\
\hline$\beta^{*}=0.7 \mathrm{~m}$ lattice: & & \\
SFPO & 0.55 & 0.36 \\
SFMO & 0.50 & 0.36 \\
SFPI & 0.33 & 0.46 \\
SFMI & 0.05 & 0.25 \\
SDPO & -0.07 & -0.44 \\
SDMO & -0.76 & -0.92 \\
SDPI & -0.75 & -0.68 \\
SDMI & -1.14 & -0.68 \\
corrected $\xi_{x, y}^{(2)}$ & $(32,-16)$ & $(330,464)$ \\
\hline$\beta^{*}=0.5 \mathrm{~m}$ lattice: & & \\
SFPO & 0.58 & 0.42 \\
SFMO & 0.62 & 0.42 \\
SFPI & 0.40 & 0.53 \\
SFMI & 0.08 & 0.31 \\
SDPO & 0.67 & -0.48 \\
SDMO & -0.31 & -1.12 \\
SDPI & -1.59 & -0.80 \\
SDMI & -1.96 & -0.80 \\
corrected $\xi_{x, y}^{(2)}$ & $(-91,-22)$ & $(341,413)$ \\
\hline \hline & & \\
\hline
\end{tabular}


Table 5: First order sextupole resonance driving terms and second order detunnings before and after the second order chromaticity correction. The proposed RHIC $100 \mathrm{GeV}$ pp run lattice with $\beta^{*}=0.7 \mathrm{~m}$ is used.

\begin{tabular}{ll}
\hline \hline \multicolumn{1}{c}{ Without $\xi^{(2)}$ correction } & \\
1st order geometric terms & $\mathrm{A}=11.93, \mathrm{phi}=-2.9$ \\
$h_{21000}$ & $\mathrm{~A}=0.72, \mathrm{phi}=147.3$ \\
$h_{30000}$ & $\mathrm{~A}=20.33, \mathrm{phi}=-48.5$ \\
$h_{10110}$ & $\mathrm{~A}=19.78, \mathrm{phi}=33.6$ \\
$h_{10020}$ & $\mathrm{~A}=2.48, \mathrm{phi}=-38.5$ \\
$h_{10200}$ & \\
Amplitude dependent tune shifts & 485.07 \\
$a_{x x}$ & 9082.27 \\
$a_{x y}$ & 221.38 \\
$a_{y y}$ & \\
\hline With $\left(^{(2)}\right.$ correction & \\
1 st order geometric terms & $\mathrm{A}=12.71, \mathrm{phi}=7.3$ \\
$h_{21000}$ & $\mathrm{~A}=0.69, \mathrm{phi}=151.5$ \\
$h_{30000}$ & $\mathrm{~A}=21.73, \mathrm{phi}=-63.5$ \\
$h_{10110}$ & $\mathrm{~A}=18.32, \mathrm{phi}=26.9$ \\
$h_{10020}$ & $\mathrm{~A}=1.44, \mathrm{phi}=-36.1$ \\
$h_{10200}$ & \\
Amplitude dependent tune shifts & \\
$a_{x x}$ & 288.81 \\
$a_{x y}$ & 9132.89 \\
$a_{y y}$ & 132.64 \\
\hline \hline
\end{tabular}

acceptable. From Table 4, the correction strengths from the Harmon module are bigger than those from the 4-knob method. The correction strengths from Harmon have a big unbalance among the 8 sextupoles families. For example, for the $\beta^{*}=0.5 \mathrm{~m}$ lattice, the biggest and the smallest required correction strengths of sextupole families from the Harmon correction are $1.96 \mathrm{~m}^{-2}$ and $0.08 \mathrm{~m}^{-2}$. While with the 4 -knob method, the biggest and the smallest required correction strengths are $1.12 \mathrm{~m}^{-2}$ and $0.31 \mathrm{~m}^{-2}$. From Table 2, SDPO is required to change polarity with the Harmon optimization. However all power supplies of RHIC chromatic sextupoles are monopoles.

Table 5 shows the first order resonance driving terms and second order detunings from sextupoles before and after the second order chromaticity correction. The proposed RHIC $100 \mathrm{GeV}$ pp run lattice with $\beta^{*}=0.7 \mathrm{~m}$ is used here. The second order chromaticities are corrected with the 4 -knob method. From Table 5 , there are only small differences in the first order resonance driving terms and second order detunings due to the sextupoles.

\section{Dynamic aperture calculation}

In this section, we calculate the $10^{6}$ turn dynamic apertures for the above lattices. The dynamic apertures are searched in the 6 angles from $15^{\circ}$ to $75^{\circ}$ with a step size of $15^{\circ}$ in the $(x / \sigma, y / \sigma)$ space. The initial transverse momenta of the particles are set to 0 . The 6-D element-by-element simplectic tracking code SixTrack [6] is used. The dynamic apertures are measured in the unit of $\sigma=\sqrt{\frac{\epsilon_{n} \beta^{*}}{6 \pi \gamma \beta}}$, where $\epsilon_{n}$ is the $95 \%$ normalized transverse beam emittance, $\gamma$ is the relativistic factor which is 106 and 266 for the $100 \mathrm{GeV}$ and $250 \mathrm{GeV}$ runs.

Two nominal working points $(28.685,29.695)$ and $(28.695,29.685)$ are used. The first order chromaticity is corrected to +1 . The second order chromaticities are corrected to below 500 with the two methods discussed above. Here the proton bunch intensity is $N_{p}=1.8 \times 10^{11}$, the proton beam emittance is $20 \pi \mathrm{mm}$.mrad. We calculate the dynamic apertures for the relative off-momentum deviation $\delta=0.0007$ and 0.0005 for the $100 \mathrm{GeV}$ and $250 \mathrm{GeV}$ lattices respectively. 


\subsection{The $100 \mathrm{GeV}$ lattices}

Table 6 shows the dynamic apertures for the proposed RHIC $2009100 \mathrm{GeV}$ pp lattices. From Table 6, for the proposed $100 \mathrm{GeV}$ lattices with the working point $(28.685,29.695)$, without second order chromaticity correction, the minimum dynamic apertures are $4.9 \sigma, 4.1 \sigma, 2.9 \sigma$ for the lattices with $\beta^{*}=0.9 \mathrm{~m}, 0.7 \mathrm{~m}$, and $0.5 \mathrm{~m}$. For the working point $(28.695,29.685)$, the minimum dynamic apertures are $4.7 \sigma, 3.6 \sigma$, and $3.6 \sigma$ for the lattices with $\beta^{*}=0.9 \mathrm{~m}, 0.7 \mathrm{~m}$, and $0.5 \mathrm{~m}$. There is a large reduction in the minimum dynamic aperture with the $\beta^{*}=0.5 \mathrm{~m}$ lattice for the working point $(28.685,29.695)$. For the working point (28.695, 29.685 ), the minimum dynamic apertures for the lattices with $\beta^{*}=0.7 \mathrm{~m}$ and $\beta^{*}=0.5 \mathrm{~m}$ both are about $3.6 \sigma$.

Then we check the dynamic aperture changes with the second order chromaticity corrections. The Harmon module and the 4-knob method both are used to do the corrections. According to Table 6, for the $100 \mathrm{GeV}$ lattices with the working point $(28.685,29.695)$, the 4-knob method yields larger minimum dynamic apertures than the Harmon module. As mentioned above in Table 4 , for the lattice with $\beta^{*}=0.5 \mathrm{~m}$, the Harmon requires the polarity reversal of SDOP family. From Table 6 , the second order chromaticity corrections improve the minimum dynamic apertures for the lattices with $\beta^{*}=0.5 \mathrm{~m}$, but reduces the minimum dynamic apertures for the lattices with $\beta^{*}=0.9 \mathrm{~m}$. Also from Table 6 , for the lattices with the working point $(28.695,29.685)$, the minimum dynamic apertures have small changes with second order chromaticity correction for the lattices with $\beta^{*}=0.7 \mathrm{~m}$ and $0.5 \mathrm{~m}$. While for the lattices with $\beta^{*}=0.9 \mathrm{~m}$, the second order chromaticity correction reduces the minimum dynamic aperture.

\subsection{The $250 \mathrm{GeV}$ lattices}

Table 7 shows the dynamic apertures for the proposed RHIC $2009250 \mathrm{GeV}$ pp lattices. From Table 7, for the proposed $250 \mathrm{GeV}$ lattices with the working point $(28.685,29.695)$, without second order chromaticity correction, the minimum dynamic apertures are $4.9 \sigma$ for both lattices with $\beta^{*}=0.7 \mathrm{~m}$ and $0.5 \mathrm{~m}$. While for the working point $(28.695,29.685)$, the minimum dynamic apertures for the lattices with $\beta^{*}=0.7 \mathrm{~m}$ and $0.5 \mathrm{~m}$ are $5.4 \sigma$ and $4.9 \sigma$. Comparing Table 6 and Table 7 , with the same $\beta^{*}=0.7 \mathrm{~m}$, without second order chromaticity correction, the $250 \mathrm{GeV}$ lattices yield $1 \sigma$ more than the $100 \mathrm{GeV}$ lattices.

From Table 7 , for the proposed $250 \mathrm{GeV}$ lattices with $\beta^{*}=0.7 \mathrm{~m}$, the second order chromaticity corrections from the 4-knob method increase the minimum dynamic aperture by more than $1 \sigma$, comparing to those without correction. However, the second order chromaticity corrections from the Harmon reduces the minimum dynamic apertures.

For the lattices with $\beta^{*}=0.5 \mathrm{~m}$, the minimum dynamic apertures with the second order chromaticity corrections from 4-knob method are comparable to that without correction. The correction strengths from the Harmon require the polarity reversal of family SDPO. However, all the RHIC sextupoles are monopoles.

\section{Dynamic apertures in the scans of beam and optics parameters}

In this section we calculate the dynamic apertures in the scans of bunch intensity and transverse beam emittance. The starting optics and beam parameters are $\beta^{*}=0.7 \mathrm{~m}$, bunch intensity is $N_{p}=1.7 \times 10^{11}$, beam emittance $20 \pi \mathrm{mm}$.mrad. The relative momentum deviation are 0.0007 and 0.0005 for $100 \mathrm{GeV}$ and $250 \mathrm{GeV}$ lattices respectively. Again in the following the dynamic apertures are given in the unit of

$\sigma=\sqrt{\frac{\epsilon_{n} \beta^{*}}{6 \gamma}}$, which differs with particle energy, $\beta^{*}$, and beam emittance. The second order chromaticities are corrected with the 4-knob method.

Table 8 lists the calculated $10^{6}$ turn dynamic apertures in the scan of bunch intensity. From Table 8 , for the $100 \mathrm{GeV}$ lattice, there is no clear trend in the minimum dynamic apertures when we scan the bunch intensity from $N_{p}=1.4 \times 10^{11}$ to $2.0 \times 10^{11}$. For the $250 \mathrm{GeV}$ lattice, we observe reduction in the minimu dynamic aperture for the cases with $N_{p}=2.0 \times 10^{11}$.

Table 9 lists the calculated $10^{6}$ turn dynamic apertures in the scan of beam emittance. From Table 9 , there is no clear trend in the minimum dynamic apertures when we scan the beam emittance from $14 \pi$ mm.mrad to $20 \pi \mathrm{mm}$.mrad. The differences in the minimum dynamic apertures are less than $0.3 \sigma$.

In all the above simulations, the $\beta$ s at the other non-collisional IPs are set to $5 \mathrm{~m}$. For the coming RHIC $250 \mathrm{GeV}$ pp run, we may set $\beta=7.5 \mathrm{~m}$ at these points. Table 10 compares the dynamic apertures for the $250 \mathrm{GeV}$ lattices with different $\beta$ s at the non-collisional IPs. The $\beta^{*}$ at IP6 and IP8 are $0.7 \mathrm{~m}$ or $0.5 \mathrm{~m}$. The second order chromaticities are corrected to below 500 with the 4 -knob method in all cases. Form Table 10 , 
Table 6: Calculated $10^{6}$ turn dynamic apertures for the proposed RHIC $2009100 \mathrm{GeV}$ pp lattices. Here bunch intensity $N_{p}=1.8 \times 10^{11}$, beam emittance $20 \pi \mathrm{mm}$.mrad, relative momentum deviation 0.0007 .

\begin{tabular}{ccccccccc}
\hline \hline Working point & $\beta^{*}$ & $\xi^{(2)}$ correction/method & $15^{\circ}$ & $30^{\circ}$ & $45^{\circ}$ & $60^{\circ}$ & $75^{\circ}$ & Minimum \\
\hline & & & & & & & & \\
$(28.685,29.695)$ & 0.9 & No & 5.7 & 5.8 & 5.4 & 4.9 & 5.4 & 4.9 \\
$(28.685,29.695)$ & 0.9 & Yes / Harmon & 6.1 & 5.9 & 5.6 & 3.6 & 4.5 & 3.6 \\
$(28.685,29.695)$ & 0.9 & Yes / 4-knob & 5.8 & 5.8 & 5.4 & 4.9 & 4.5 & 4.5 \\
& & & & & & & & \\
$(28.685,29.695)$ & 0.7 & No & 4.7 & 4.7 & 4.5 & 4.1 & 4.2 & 4.1 \\
$(28.685,29.695)$ & 0.7 & Yes / Harmon & 4.9 & 4.9 & 4.7 & 3.9 & 3.5 & 3.5 \\
$(28.685,29.695)$ & 0.7 & Yes / 4-knob & 5.1 & 5.2 & 4.5 & 4.2 & 4.1 & 4.1 \\
& & & & & & & \\
$(28.685,29.695)$ & 0.5 & No & 3.9 & 3.6 & 3.4 & 3.0 & 2.9 & 2.9 \\
$(28.685,29.695)$ & 0.5 & Yes / Harmon & 3.9 & 4.1 & 3.6 & 3.4 & 4.3 & 3.4 \\
$(28.685,29.695)$ & 0.5 & Yes / 4-knob & 4.4 & 4.3 & 3.9 & 3.6 & 3.5 & 3.5 \\
& & & & & & & \\
\hline & & & & & & & \\
$(28.695,29.685)$ & 0.9 & No & 4.7 & 5.1 & 5.8 & 5.7 & 5.9 & 4.7 \\
$(28.695,29.685)$ & 0.9 & Yes / Harmon & 4.3 & 4.5 & 5.6 & 5.7 & 6.0 & 4.3 \\
$(28.695,29.685)$ & 0.9 & Yes / 4-knob & 4.2 & 4.5 & 5.8 & 5.9 & 6.2 & 4.2 \\
& & & & & & & \\
$(28.695,29.685)$ & 0.7 & No & 3.6 & 4.5 & 4.8 & 5.3 & 5.3 & 3.6 \\
$(28.695,29.685)$ & 0.7 & Yes / Harmon & 3.6 & 4.2 & 4.9 & 5.1 & 5.3 & 3.6 \\
$(28.695,29.685)$ & 0.7 & Yes / 4-knob & 3.8 & 3.8 & 5.2 & 5.3 & 5.5 & 3.8 \\
& & & & & & & & \\
$(28.695,29.685)$ & 0.5 & Yes / Harmon & 3.6 & 3.8 & 4.1 & 4.2 & 4.2 & 3.6 \\
$(28.695,29.685)$ & 0.5 & Yes / 4-knob & 3.6 & 4.1 & 4.9 & 4.4 & 4.1 & 3.6 \\
$(28.695,29.685)$ & 0.5 & Yes & 4.6 & 4.6 & 3.6 \\
\hline \hline
\end{tabular}

Table 7: Calculated $10^{6}$ turn dynamic apertures for the proposed RHIC $2009250 \mathrm{GeV}$ pp lattices. Here bunch intensity $N_{p}=1.8 \times 10^{11}$, beam emittance $20 \pi \mathrm{mm}$.mrad, relative momentum deviation 0.0005 .

\begin{tabular}{ccccccccc}
\hline \hline working point & $\beta^{*}$ & $\xi^{(2)}$ correction/method & $15^{\circ}$ & $30^{\circ}$ & $45^{\circ}$ & $60^{\circ}$ & $75^{\circ}$ & Minimum \\
\hline & & No & 6.5 & 6.2 & 5.6 & 5.1 & 4.9 & 4.9 \\
$(28.685,29.695)$ & 0.7 & Yes / Harmon & 6.5 & 5.7 & 5.7 & 5.2 & 3.9 & 3.9 \\
$(28.685,29.695)$ & 0.7 & Yes / 4-knob & 6.3 & 6.5 & 6.0 & 5.9 & 6.4 & 6.0 \\
$(28.685,29.695)$ & 0.7 & & & & & & & \\
& & No & 6.2 & 6.7 & 6.6 & 4.9 & 5.3 & 4.9 \\
$(28.685,29.695)$ & 0.5 & Yes / Harmon & 6.8 & 6.6 & 6.3 & 4.8 & 4.1 & 4.1 \\
$(28.685,29.695)$ & 0.5 & Yes / 4-knob & 6.5 & 6.6 & 6.6 & 4.9 & 4.8 & 4.8 \\
$(28.685,29.695)$ & 0.5 & & & & & & & \\
& & & & & & & & \\
& & & & & & \\
$(28.695,29.685)$ & 0.7 & No & 5.4 & 5.6 & 5.7 & 7.3 & 5.4 \\
$(28.695,29.685)$ & 0.7 & Yes / Harmon & 4.8 & 4.9 & 6.6 & 6.9 & 4.8 \\
$(28.695,29.685)$ & 0.7 & Yes / 4-knob & 7.9 & 7.2 & 6.9 & 6.8 & 7.3 & 6.8 \\
& & & & & & & \\
$(28.695,29.685)$ & 0.5 & No & 4.9 & 5.1 & 5.8 & 6.3 & 7.1 & 4.9 \\
$(28.695,29.685)$ & 0.5 & Yes / Harmon & 5.5 & 5.6 & 5.9 & 6.4 & 7.6 & 5.5 \\
$(28.695,29.685)$ & 0.5 & Yes / 4-knob & 7.5 & 4.9 & 6.3 & 6.7 & 7.2 & 4.9 \\
\hline \hline
\end{tabular}


Table 8: Calculated $10^{6}$ turn dynamic apertures in the scan of bunch intensity. Here $\beta^{*}=0.7 \mathrm{~m}$, bunch intensity $1.7 \times 10^{11}$, relative momentum deviation are 0.0007 and 0.0005 for $100 \mathrm{GeV}$ and $250 \mathrm{GeV}$ lattices respectively.

\begin{tabular}{cccccccc}
\hline \hline working point & Bunch intensity & $15^{\circ}$ & $30^{\circ}$ & $45^{\circ}$ & $60^{\circ}$ & $75^{\circ}$ & Minimum \\
\hline $100 \mathrm{GeV}:$ & & & & & & & \\
& & & & & & & \\
$(28.685,29.695)$ & $1.4 \times 10^{11}$ & 4.9 & 5.1 & 4.9 & 4.5 & 4.1 & 4.1 \\
$(28.685,29.695)$ & $1.7 \times 10^{11}$ & 5.1 & 5.1 & 4.8 & 4.1 & 4.3 & 4.1 \\
$(28.685,29.695)$ & $2.0 \times 10^{11}$ & 4.9 & 5.1 & 4.7 & 4.2 & 3.9 & 3.9 \\
& & & & & & & \\
$(28.695,29.685)$ & $1.4 \times 10^{11}$ & 3.5 & 4.1 & 4.9 & 5.4 & 5.6 & 3.5 \\
$(28.695,29.685)$ & $1.7 \times 10^{11}$ & 3.8 & 3.9 & 5.3 & 5.6 & 5.6 & 3.8 \\
$(28.695,29.685)$ & $2.0 \times 10^{11}$ & 3.8 & 3.9 & 5.1 & 5.5 & 4.9 & 3.8 \\
& & & & & & & \\
\hline $250 \mathrm{GeV}:$ & & & & & & & \\
$(28.685,29.695)$ & $1.4 \times 10^{11}$ & 6.1 & 6.9 & 6.5 & 6.1 & 6.3 & 6.1 \\
$(28.685,29.695)$ & $1.7 \times 10^{11}$ & 6.5 & 6.7 & 6.1 & 6.1 & 6.4 & 6.1 \\
$(28.685,29.695)$ & $2.0 \times 10^{11}$ & 8.8 & 6.7 & 5.7 & 5.1 & 6.5 & 5.7 \\
& & & & & & & \\
$(28.695,29.685)$ & $1.4 \times 10^{11}$ & 7.8 & 7.5 & 7.1 & 7.1 & 7.3 & 7.1 \\
$(28.695,29.685)$ & $1.7 \times 10^{11}$ & 6.7 & 7.7 & 6.9 & 6.7 & 7.2 & 6.9 \\
$(28.695,29.685)$ & $2.0 \times 10^{11}$ & 6.8 & 6.2 & 6.6 & 6.7 & 6.7 & 6.2 \\
\hline \hline
\end{tabular}

for the working point $(28.685,29.695)$, the lattices with $\beta=7.5 \mathrm{~m}$ give higher minimum dynamic apertures. For the working point $(28.695,29.685)$, the lattices with $\beta=7.5 \mathrm{~m}$ do not.

\section{Effect of $\xi^{(2)}$ correction on beam lifetime}

In this section, we evaluate the beam lifetime with the second order chromaticity correction. To do that, we track 6400 macro-particles up to $2 \times 10^{6}$ turns [7]. These macro-particles are sampled from a hollow Gaussian distribution from 3-6 $\sigma$ in the $(x / \sigma, y / \sigma)$ plane. The 6400 macro-particles actually represent 100348 macroparticles in a Gaussian distribution bunch. Particles below $3 \sigma$ are assumed live after $2 \times 10^{6}$ turn tracking. In the initial particle distribution generation, we used rms bunch size $\left(\sigma_{l}\right)_{r m s}=0.454 \mathrm{~m}$ and rms relative energy spread $\left(\frac{d p}{p_{0}}\right)_{r m s}=1.414 \times 10^{-4}$.

The proposed $100 \mathrm{GeV}$ Blue ring lattice with $\beta^{*}=0.7 \mathrm{~m}$ is used in this section. The bunch intensity $N_{p}=1.7 \times 10^{11}$ and transverse beam emittance $\epsilon_{n}=20 \pi \mathrm{mm}$.mrad. Figure 1 shows the relative beam intensities for the cases without and with second order chromaticity correction. The same initial distribution of macro-particles are used for the two cases. Here the second chromaticities are corrected with the Harmon module. The IR nonlinearities are included. From Figure 1, the particle loss rates are the same in the $2 \times 10^{6}$ turns, or in about 24 seconds after cogging. The beam decay from the trackings is about $20 \% /$ hour for both cases without and with second order chromaticities correction.

Then we exclude the IR nonlinearities to do the above comparison. The second order chromaticity correction are also corrected with the Harmon. From Figure 2, the slope of particle loss is almost the same for cases without and with second order chromaticities. The beam decay from the tracking is about $8 \% /$ hour for both cases without and with second order chromaticities correction.

In the simulation, the beam emittance growth rate is not comparied due to the large fluctuations in the trackings. To get meaningful emittance information, we need dramatically increase the numbers of tracking particles and tracking turns, which in return requires a huge amount of CPU computation time. 
Table 9: Calculated $10^{6}$ turn dynamic apertures in the scan of beam emittance. Here $\beta^{*}=0.7 \mathrm{~m}$, bunch intensity $1.7 \times 10^{11}$, relative momentum deviation are 0.0007 and 0.0005 for $100 \mathrm{GeV}$ and $250 \mathrm{GeV}$ lattices respectively.

\begin{tabular}{cccccccc}
\hline \hline working point & emittance & $15^{\circ}$ & $30^{\circ}$ & $45^{\circ}$ & $60^{\circ}$ & $75^{\circ}$ & Minimum \\
\hline $100 \mathrm{GeV}:$ & & & & & & & \\
& & & & & & & \\
$(28.685,29.695)$ & $14 \pi \mathrm{mm} . \mathrm{mrad}$ & 5.8 & 5.7 & 4.9 & 4.6 & 4.1 & 4.1 \\
$(28.685,29.695)$ & $17 \pi \mathrm{mm} . \mathrm{mrad}$ & 5.3 & 5.2 & 4.7 & 4.3 & 4.5 & 4.3 \\
$(28.685,29.695)$ & $20 \pi \mathrm{mm} . \mathrm{mrad}$ & 5.1 & 5.1 & 4.8 & 4.1 & 4.3 & 4.1 \\
& & & & & & & \\
$(28.695,29.685)$ & $14 \pi \mathrm{mm} . \mathrm{mrad}$ & 3.8 & 3.9 & 4.9 & 5.9 & 5.7 & 3.8 \\
$(28.695,29.685)$ & $17 \pi \mathrm{mm} . \mathrm{mrad}$ & 3.8 & 4.3 & 5.4 & 5.7 & 5.7 & 3.8 \\
$(28.695,29.685)$ & $20 \pi \mathrm{mm} . \mathrm{mrad}$ & 3.8 & 3.9 & 5.3 & 5.6 & 5.6 & 3.8 \\
\hline $250 \mathrm{GeV}:$ & & & & & & & \\
& & & & & & & \\
$(28.685,29.695)$ & $14 \pi \mathrm{mm} . \mathrm{mrad}$ & 6.9 & 7.1 & 6.7 & 6.3 & 6.8 & 6.3 \\
$(28.685,29.695)$ & $17 \pi \mathrm{mm} . \mathrm{mrad}$ & 6.9 & 6.8 & 6.5 & 6.4 & 6.9 & 6.4 \\
$(28.685,29.695)$ & $20 \pi \mathrm{mm} . \mathrm{mrad}$ & 6.5 & 6.7 & 6.1 & 6.1 & 6.4 & 6.1 \\
& & & & & & & \\
$(28.695,29.685)$ & $14 \pi \mathrm{mm} . \mathrm{mrad}$ & 8.0 & 7.7 & 6.8 & 6.6 & 8.6 & 6.6 \\
$(28.695,29.685)$ & $17 \pi \mathrm{mm} . \mathrm{mrad}$ & 7.2 & 7.1 & 6.8 & 6.9 & 7.8 & 6.8 \\
$(28.695,29.685)$ & $20 \pi \mathrm{mm} . \mathrm{mrad}$ & 6.7 & 7.7 & 6.9 & 6.7 & 7.2 & 6.9 \\
\hline \hline
\end{tabular}

Table 10: Calculated $10^{6}$ turn dynamic apertures for the $250 \mathrm{GeV}$ lattices with different $\beta$ functions at the non-collisional IPs. Bunch intensity $1.8 \times 10^{11}$, beam emittance $20 \pi \mathrm{mm}$.mrad, relative momentum deviation are 0.0005 .

\begin{tabular}{ccccccccc}
\hline \hline working point & $\beta *$ & $\beta$ s at other IPs & $15^{\circ}$ & $30^{\circ}$ & $45^{\circ}$ & $60^{\circ}$ & $75^{\circ}$ & Minimum \\
\hline Above diagonal: & & & & & & & & \\
& & & & & & & & \\
$(28.685,29.695)$ & $0.7 \mathrm{~m}$ & $5.0 \mathrm{~m}$ & 6.3 & 6.5 & 6.0 & 5.9 & 6.4 & 6.0 \\
$(28.685,29.695)$ & $0.7 \mathrm{~m}$ & $7.5 \mathrm{~m}$ & 7.9 & 8.4 & 7.4 & 7.1 & 6.1 & 6.1 \\
& & & & & & & & \\
$(28.685,29.695)$ & $0.5 \mathrm{~m}$ & $5.0 \mathrm{~m}$ & 6.5 & 6.6 & 6.6 & 4.9 & 4.8 & 4.8 \\
$(28.685,29.695)$ & $0.5 \mathrm{~m}$ & $7.5 \mathrm{~m}$ & 6.6 & 6.2 & 6.3 & 5.8 & 5.6 & 5.6 \\
& & & & & & & & \\
\hline Below diagonal: & & & & & & & & \\
& & & & & & & & \\
$(28.695,29.685)$ & $0.7 \mathrm{~m}$ & $5.0 \mathrm{~m}$ & 7.9 & 7.2 & 6.9 & 6.8 & 7.3 & 6.8 \\
$(28.695,29.685)$ & $0.7 \mathrm{~m}$ & $7.5 \mathrm{~m}$ & 8.0 & 6.5 & 7.7 & 6.9 & 7.8 & 6.5 \\
& & & & & & & & \\
$(28.695,29.685)$ & $0.5 \mathrm{~m}$ & $5.0 \mathrm{~m}$ & 7.5 & 4.9 & 6.3 & 6.7 & 7.2 & 4.9 \\
$(28.695,29.685)$ & $0.5 \mathrm{~m}$ & $7.5 \mathrm{~m}$ & 5.3 & 4.9 & 5.7 & 6.6 & 6.8 & 4.9 \\
\hline \hline
\end{tabular}




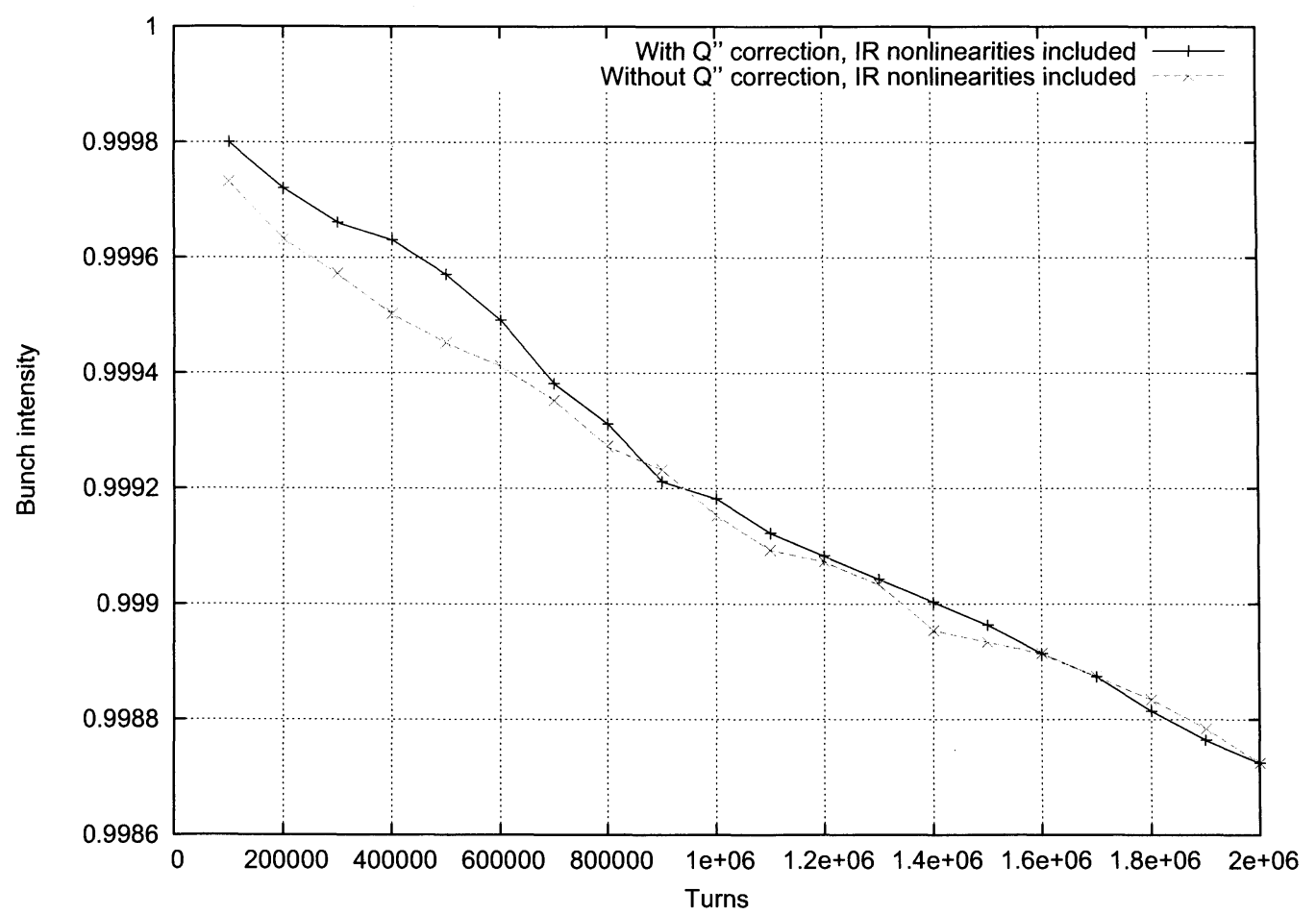

Figure 1: Comparison of beam lifetime without and with second order chromaticity correction. Here IR nonlinear errors are included.

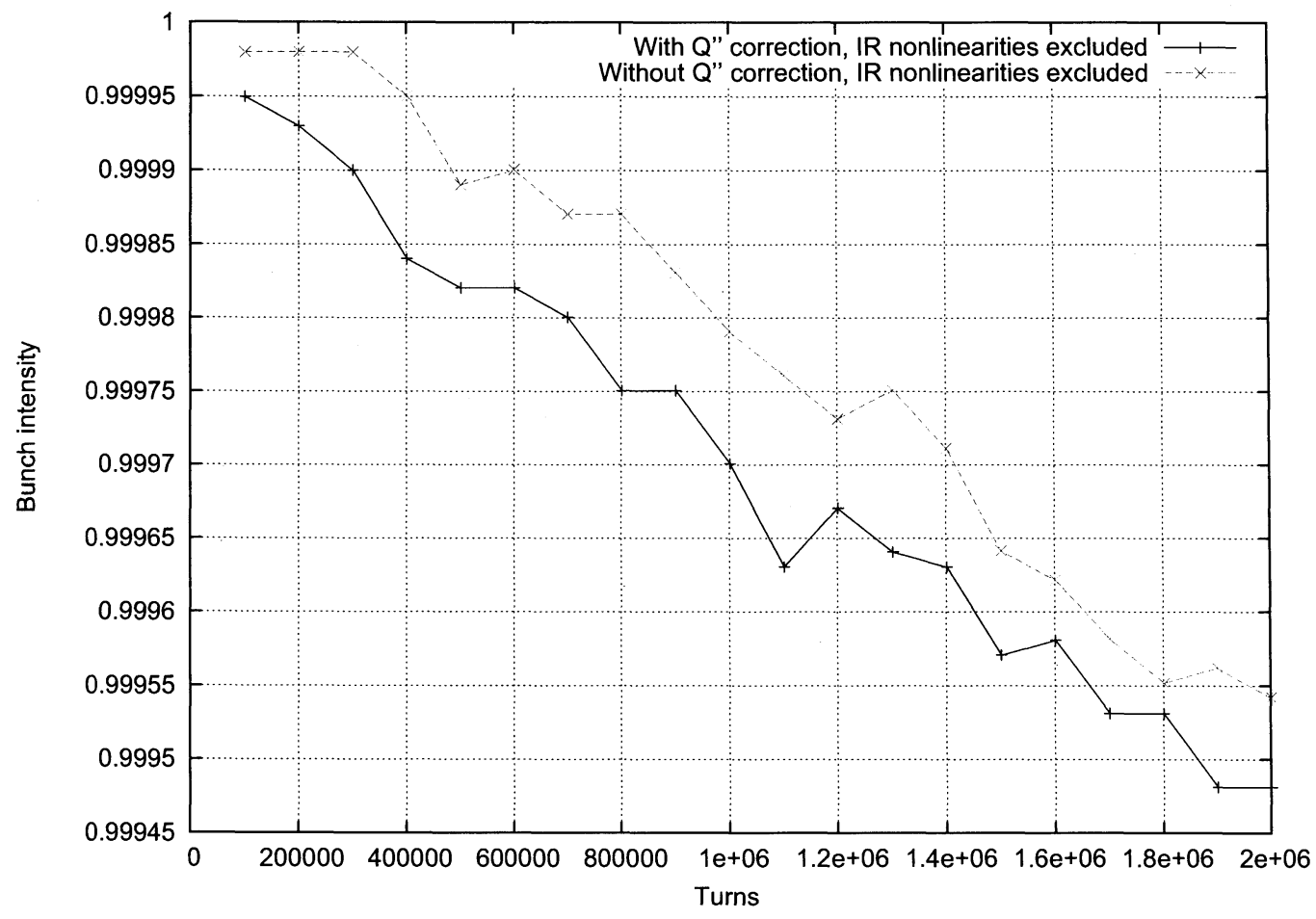

Figure 2: Comparison of beam lifetime without and with second order chromaticity correction. Here IR nonlinear errors are excluded. 


\section{Conclusion}

We evaluated the dynamic apertures with the proposed RHIC lattices for the 2009 polarized proton runs. The $100 \mathrm{GeV}$ lattices with $\beta^{*}=0.9 \mathrm{~m}, 0.7 \mathrm{~m}$, and $0.5 \mathrm{~m}$, and the $250 \mathrm{GeV}$ lattices with $\beta^{*}=0.7 \mathrm{~m}$ and $0.5 \mathrm{~m}$ are all checked. For each lattice, the second order chromaticities are corrected with the Harmon module and the 4-knob method.

For the proposed $100 \mathrm{GeV}$ lattices, for the working point $(28.685,29.695)$, without the second order chromaticity correction, the minimum dynamic apertures are about $4.9 \sigma, 4: 1 \sigma$, and $2.9 \sigma$ for the lattices with $\beta^{*}=0.9 \mathrm{~m}, 0.7 \mathrm{~m}$, and $0.5 \mathrm{~m}$ respectively. For the working point $(28.695,29.685)$, these values are about $4.7 \sigma, 3.6 \sigma$ and $3.6 \sigma$ respectively. For the proposed $250 \mathrm{GeV}$ lattices, for the lattices with $\beta^{*}=0.7 \mathrm{~m}$, the minimum dynamic apertures are around $5 \sigma$ which is about $1 \sigma$ larger than the same $\beta^{*}$ lattice at $100 \mathrm{GeV}$.

For $250 \mathrm{GeV}$ lattices, the dynamic aperture did not drop much when the $\beta^{*}$ is squeezed from $0.7 \mathrm{~m}$ to $0.5 \mathrm{~m}$. For the $100 \mathrm{GeV}$ lattices, the working point $(28.685,29.695)$ provides slightly bigger minimum dynamic aperture than the working point $(28.695,29.685)$, while for the $250 \mathrm{GeV}$ lattices, the working point $(28.695,29.685)$ shows slightly bigger minimum dynamic aperture.

Values of the minimum dynamic apertures show that the second order chromaticity plays an important role for the lower $\beta^{*}$ lattices. For the working point $(28.685,29.695)$, the second order chromaticity correction is needed for lattices with $\beta^{*}=0.7 \mathrm{~m}$ and $0.5 \mathrm{~m}$. For the working point $(28.695,29.685)$, there is no difference in the minimum dynamic apertures without or with second order chromaticity correction. Most of the time, the second order chromaticity correction strengths from the 4-knob method provides larger minimum dynamic apertures than those from the Harmon module. The 4-knob method reduces the unbalance in the correction strengths among the sextupole families and avoids the polarity reversals of sextupoles.

We also compared the dynamic apertures with respect to the bunch intensity and transverse beam emittance. For the $100 \mathrm{GeV}$ lattice with $\beta^{*}=0.7 \mathrm{~m}$, the differences in the minimum dynamic apertures are less than $0.4 \sigma$ when we scan the bunch intensity from $1.4 \times 10^{11}$ to $2.0 \times 10^{11}$ and when we scan the beam emittance from $14 \pi \mathrm{mm}$.mrad to $20 \pi \mathrm{mm}$.mrad. For the $250 \mathrm{GeV}$ lattices with $\beta^{*}=0.7 \mathrm{~m}$, for the working point $(28.685,29.695)$, lattices with $\beta=7.5 \mathrm{~m}$ at other non-collisional IPs provided higher minimum dynamic apertures than that with $\beta=5 \mathrm{~m}$, while for the working point $(28.695,29.685)$, the lattices with $\beta=7.5 \mathrm{~m}$ do not.

In the end, we compared the simulated beam lifetime without and with second order chromaticity correction in the $2 \times 10^{6}$ turn trackings with 6400 macro-particles. For the $100 \mathrm{GeV}$ lattice with $\beta^{*}=0.7 \mathrm{~m}$, there is no difference in the beam lifetime in $2 \times 10^{6}$ turns for the cases with or without the second order chromaticity correction. The effect of second order chromaticity correction on the emittance growth is not checked. The second order chromaticity correction may mitigate the beam-beam effects when the bunch intensity is further increased or the beam emittance is further reduced.

\section{References}

[1] Y. Luo, et al., Dynamic Aperture evaluation at the current working point for RHIC polarized proton run, BNL C-AD AP Note 271. Available at http://www.cadops.bnl.gov/AP/ap_notes/cad_ap_index.html.

[2] Y. Luo and W. Fischer, Outline of using an electron lens for the RHIC head-on beam-beam compensation, BNL C-AD AP Note 286. Available at http://www.cadops.bnl.gov/AP/ap_notes/cad_ap_index.html.

[3] S. Tepikian, Nonlinear chromaticity correction, 2006 RHIC Retreat, July 10-13, 2006, Port Jefferson, NY. Available at http://www.c-ad.bnl.gov/RHIC/Retreat2006/agenda.html.

[4] Y. Luo, et al., Sorting chromatic sextupoles for easily and effectively correcting second order chromaticity in the Relativistic Heavy Ion Collider, to be released.

[5] W. Fischer, Beam-beam and BTF, 2006 RHIC Accelerator Physics Experiments Workshop, November 2-3, 2005, BNL. Available at http://www.c-ad.bnl.gov/APEX/APEXWorkshop2006/agenda.htm.

[6] SixTrack code homepage, http://frs.home.cern.ch/frs/.

[7] Y. Luo, et al., Multi-Particle Weak-Strong Simulations of RHIC Head-on Beam-Beam Compensation, in the Proceedings of EPAC 08, Genoa, Italy. 